\title{
Development of a Discriminating In Vitro Dissolution Method for the Poorly Soluble Drug Rimonabant: Effect of Formulation Variables on Dosage Form Release Profiles
}

Felipe K. Hurtado, Aline Ravanello, Bruna G. S. Torres, Gabriele D. Souto, Ruy Carlos R. Beck, and Clarice M. B. Rolim*

Programa de Pós-Graduação em Ciências Farmacêuticas, Universidade Federal de Santa Maria, Av. Roraima 1000, 97105-900 Santa Maria, RS, Brazil

\begin{abstract}
The effect of formulation parameters on the in vitro release profile of a poorly soluble drug was investigated using rimonabant as model drug. A dissolution test was developed to evaluate the release profile of rimonabant from both 20$\mathrm{mg}$ film-coated tablet and capsule dosage forms. The test was applied to compare dissolution profiles of different dosage forms and to evaluate the effect of formulation parameters on the in vitro release profile of the drug. Four different commercially available products were evaluated, and the results obtained show very distinct rates and extent of dissolution among them. The type of excipients used in the capsule formulation significantly influenced the dissolution rate of the formulations studied.
\end{abstract}

\section{INTRODUCTION}

M any drugs are poorly soluble or insoluble in water, which results in poor bioavailability because the solubility of a drug is an important factor in determining the rate and extent of its absorption (1). Drug release rate is one of the most important parameters for solid oral drug delivery systems; therefore, the therapeutic response is a function of the concentration of drug available to be absorbed and reach the bloodstream (2). Physical factors important to drug dissolution include particle size, molecular size, hydrophilicity, and crystalline structure. The volume of medium into which the drug must dissolve can also play a role in determining the dissolution rate (3).

Physical modifications often aim to increase the surface area, solubility, and wettability of the powder particles and, therefore, typically focus on particle size reduction or generation of amorphous states. Classically, particle size reduction is performed by milling, and a wide variety of apparatus is available. The increase in bioavailability after micronization of drugs is well known, and the technique has been applied to a variety of poorly water-soluble compounds (4). Another approach for improving solubility is the complexation and incorporation of drugs into cyclodextrins (5).

The incorporation of adjuvants (e.g., diluents, lubricants, and surfactants) into the formulation of a solid oral dosage form can cause significant effects on the dissolution rate of drugs, especially those that are hydrophobic and poorly

*Corresponding author. Current address: Departamento de Farmácia Industrial, Universidade Federal de Santa Maria, Av. Roraima 1000, 97105900 Santa Maria, RS, Brazil. soluble (6). In the case of Class 2 drugs in the Biopharmaceutics Classification System (BCS), dissolution may be the ratelimiting step for drug absorption, so suitable dissolution tests can be used to predict differences in bioavailability among different formulations (7). The choice of formulation is often of critical importance in establishing a successful product for oral administration of this class of drugs (8).

In this context, the purpose of the present study was to evaluate and compare the dissolution profiles of several compounded formulations to that of a reference product using rimonabant as a model drug. This drug is poorly soluble in water and has high in vitro permeability; it is therefore classified as BCS Class 2 (9). To date, there is no published dissolution test for the evaluation of in vitro release profiles of this drug from immediate-release solid oral dosage forms. Therefore, we also developed a dissolution method for rimonabant to determine its release profiles from pharmaceutical formulations. We prepared several formulations containing different types and proportions of excipients and variations in drug particle size to assess the influence of formulation and drug physical properties on the dissolution kinetics of the drug

\section{MATERIALS AND METHODS}

\section{Chemicals}

Rimonabant reference substance $(99.5 \%)$ was purchased from Sequoia Research Products (Pangbourne, UK). Acomplia (Sanofi-Aventis, Paris, France) film-coated tablets and compounded capsules containing $20 \mathrm{mg}$ of rimonabant were obtained from commercial sources. Gradient grade LiChrosolv acetonitrile and methanol were 
Table 1. Composition of Formulations Prepared for In Vitro Release Study

\begin{tabular}{|c|c|c|c|c|c|c|c|c|c|c|}
\hline \multirow[b]{3}{*}{ Ingredient } & \multicolumn{10}{|c|}{ Amount per capsule ${ }^{a}$} \\
\hline & \multicolumn{2}{|c|}{ Formulation 1} & \multicolumn{2}{|c|}{ Formulation 2} & \multicolumn{2}{|c|}{ Formulation 3} & \multicolumn{2}{|c|}{ Formulation 4} & \multicolumn{2}{|c|}{ Formulation $5^{b}$} \\
\hline & mg & $\%$ & mg & $\%$ & mg & $\%$ & mg & $\%$ & mg & $\%$ \\
\hline Rimonabant, hydrochloride & 20 & 6.67 & 20 & 6.67 & 20 & 6.67 & 20 & 6.67 & 20 & 6.67 \\
\hline Starch & 274 & 91.33 & 268 & 89.33 & 45 & 15.00 & 45 & 15.00 & 45 & 15.00 \\
\hline Lactose & - & - & - & - & 115 & 38.33 & 115 & 38.33 & 115 & 38.33 \\
\hline Povidone K 30 & - & - & - & - & 15 & 5.00 & 15 & 5.00 & 15 & 5.00 \\
\hline Croscarmellose sodium & - & - & - & - & 30 & 10.00 & 30 & 10.00 & 30 & 10.00 \\
\hline Sodium lauryl sulfate & - & - & 6 & 2.00 & 6 & 2.00 & - & - & 6 & 2.00 \\
\hline Microcrystalline cellulose & - & - & - & - & 68.25 & 22.75 & 74.25 & 24.75 & 68.25 & 22.75 \\
\hline Magnesium stearate & - & - & - & - & 0.75 & 0.25 & 0.75 & 0.25 & 0.75 & 0.25 \\
\hline Colloidal silicon dioxide & 6 & 2.00 & 6 & 2.00 & - & - & - & - & - & - \\
\hline
\end{tabular}

purchased from Merck (Darmstadt, Germany). Sodium lauryl sulfate (SLS) was purchased from Vetec (Rio de Janeiro, Brazil). Water was purified with a WaterPro PS, Labconco system (Missouri, USA). All other chemicals were of pharmaceutical grade. The phosphate and acetate USP buffers ( $\mathrm{pH} 6.8$ and 4.5, respectively) were prepared as described in USP 32 (14). In the present study, four commercial formulations of rimonabant $20 \mathrm{mg}$ were employed:

- Reference product (Acomplia, film-coated tablet): starch, lactose monohydrate, povidone $\mathrm{K} 30$, croscarmellose sodium, sodium lauryl sulfate, microcrystalline cellulose, magnesium stearate, hypromellose $15 \mathrm{mPa}$.s, titanium dioxide, macrogol 3000, and carnauba wax.

- Product A (capsule): starch and Aerosil (colloidal silicon dioxide).

- Product B (capsule): starch, sodium lauryl sulfate, and microcrystalline cellulose.

- Product C (capsule): lactose, magnesium stearate, microcrystalline cellulose, Aerosil, HPMC (hydroxypropyl methylcellulose), and Explotab (sodium starch glycolate and sodium carboxymethyl starch).

\section{Preparation of Hard Gelatin Capsules}

Rimonabant capsules containing different excipients were prepared to evaluate the effect of changes in formulation parameters on in vitro dissolution of the drug and the discriminating capacity of the dissolution method. The influence of capsule diluent (e.g., starch, lactose, microcrystalline cellulose), disintegrants (e.g., crospovidone, croscarmellose sodium), and SLS, an anionic surfactant, was studied. Formulations were prepared by physically mixing rimonabant (6.67\%) with excipients for $10 \mathrm{~min}$ and then manually filling the powder mixture into hard gelatin capsules. Also studied was the effect of particle size on the dissolution rate of rimonabant. Micronization was accomplished by milling the drug for $30 \mathrm{~min}$ and mixing it with excipients. Rimonabant raw drug was crushed to a fine powder using a mortar and pestle. Particle size analysis was determined by optical microscopy at 200x. The composition of capsule formulations is shown in Table 1.

\section{In Vitro Dissolution Study}

The dissolution test was performed in a PHARMA TEST multi-bath $(n=6)$ dissolution system (Hamburg, Germany). The assay was performed using USP Apparatus 2 (paddle) at $50 \mathrm{rpm}$ and $900 \mathrm{~mL}$ of medium at $37 \pm 0.5^{\circ} \mathrm{C}$. The dissolution medium consisted of $0.15 \%(\mathrm{w} / \mathrm{v}) \mathrm{SLS}$ in water. Each dissolution test was performed with twelve units of the dosage form. Sample aliquots $(10 \mathrm{~mL})$ were withdrawn at $5,10,15,20,30,45$, and 60 min and replaced with an equal volume of the fresh medium at $37^{\circ} \mathrm{C}$ to maintain a constant total volume. Spiral stainless steel capsule sinkers (SOTAX, Switzerland) were used to prevent floating of the capsules. Each sample was filtered through quantitative filter paper (Schleicher \& Schuell, Germany), appropriately diluted with mobile phase, and filtered through a $0.45-\mu \mathrm{m}$ polyamide membrane filter (Sartorius, Germany) before HPLC analysis. All assays and measurements were performed in a dark room to avoid degradation of the drug due to its reported low photostability (10).

Different dissolution media and rotation speeds were tested to find the best conditions for evaluating the dissolution rate of rimonabant. Initial testing was carried out using three units of the reference product (Acomplia) in each of four media: $\mathrm{pH} 6.8$ phosphate buffer, $\mathrm{pH} 4.5$ acetate buffer, $0.1 \mathrm{M}$ hydrochloric acid, and $0.5 \%(\mathrm{w} / \mathrm{v}) \mathrm{SLS}$ in water. The volume of the medium was maintained at $900 \mathrm{~mL}$, and the paddle method $(50 \mathrm{rpm})$ was used. The concentration of SLS in dissolution medium, type of apparatus (paddle or basket), and rotation speed were optimized to obtain a discriminating ability of the test method. For BCS Class 2 compounds, in vitro dissolution testing can be predictive 
Table 2. Calculation of the Dissolution Efficiency and Applied Mathematical Models to the Dissolution Data of Rimonabant FilmCoated Tablets and Capsules

\begin{tabular}{|c|c|c|}
\hline Approach & Method & Equation \\
\hline Model-independent & Dissolution efficiency (DE) & $D E \%=A U C_{0-60} / A U C_{\mathrm{TR}} \times 100$ \\
\hline \multirow{3}{*}{ Model-dependent } & Zero-order & $\%$ dissol $=k t$ \\
\hline & Mono-exponential & $\%$ dissol $=100\left(1-\mathrm{e}^{-k t}\right)$ \\
\hline & Bi-exponential & $\%$ dissol $=100\left[1-\left(A e^{-k 1 t}+B e^{-k 2 t}\right)\right]$ \\
\hline
\end{tabular}

$A \cup C_{0-60}: \quad$ area under the dissolution curve from 0 to $60 \mathrm{~min}$

$A \cup C_{\mathrm{TR}}: \quad$ total area of the rectangle at the same time

$\%$ dissol: percent dissolved at time $t$

$k_{1}$ and $k_{2}$ : dissolution rate constants

of in vivo performance since absorption is solubility limited (11). When evaluating the concentration of surfactant, the aim is to use the lowest amount needed to solubilize the drug substance in the dosage form to achieve greater than $85 \%$ dissolution in a reasonable amount of time $(12,13)$.

\section{HPLC Analysis}

Rimonabant was analyzed using a previously validated HPLC method (10). Briefly, analysis of dissolution samples was performed on a Shimadzu HPLC system (Kyoto, Japan) equipped with a CBM-20A system controller, LC-20AT quaternary pump, DGU-20A5 on-line degasser, SIL-20A autosampler, and SPD-M20A diode array detector. Analytical separation was performed on a Gemini $C_{18}$ column $(150 \times 4.6 \mathrm{~mm}$ i.d., $5-\mu \mathrm{m}$ particle size) coupled to $\mathrm{a} \mathrm{C}_{18}$ guard cartridge $(4 \times 3.0 \mathrm{~mm}$ i.d.) from Phenomenex (Torrance, USA). The isocratic mobile phase was a mixture of acetonitrile/water (75:25, v/v) without $\mathrm{pH}$ adjustment and was pumped at a flow rate of $1.0 \mathrm{~mL} / \mathrm{min}$. The injection volume was $20 \mu \mathrm{L}$. Detection was achieved at $215 \mathrm{~nm}$. Data integration was performed using Shimadzu LCsolution software. The analytical curves were constructed by plotting concentration versus peak area and showed linearity in the $0.5-50 \mu \mathrm{g} / \mathrm{mL}$ range.

The method was validated by the evaluation of the following parameters: specificity, linearity, precision, and accuracy, according to the $\mathrm{ICH}$ guidelines for validation of analytical procedures (14). The specificity of the analytical method was evaluated by preparing a placebo sample of the reference tablet formulation. The placebo consisted of all the excipients and hard gelatin capsule without the active ingredient. The concentration of excipients in the formulation was based on the literature (15) and calculated for an average tablet weight of $312.6 \mathrm{mg}(n=10)$. The placebo sample was transferred to the vessel, which was filled with $900 \mathrm{~mL}$ of dissolution medium at $37 \pm$ $0.5^{\circ} \mathrm{C}$ and stirred for $60 \mathrm{~min}$ using paddle. Aliquots were withdrawn and analyzed by HPLC.

\section{Drug Stability}

Solution stability is an important aspect to be considered when selecting the dissolution medium (16). The stability of samples in dissolution medium was evaluated by leaving them in the dissolution vessels at room temperature and protected from light for $24 \mathrm{~h}$. The assay was performed in triplicate. Samples were considered stable when the content of drug in solution was between $98 \%$ and $102 \%$ of the initial value.

\section{Analysis of Dissolution Profiles}

Mathematical modeling of dissolution profiles (Acomplia and products $A, B$, and $C$ ) was performed using Scientist v2.01 software (MicroMath, Salt Lake City, USA). To understand the kinetics of drug release from the dosage forms, both mono-exponential and bi-exponential models were applied to verify which model better fit the dissolution data (17). Parameters calculated were $k$ (a kinetic constant measuring the velocity of drug release), $A, B, r$ (correlation coefficient), and MSC (model selection criteria). The dissolution profiles were also compared by the model-independent method, dissolution efficiency $(D E)$. DE was calculated from the area under the dissolution curve at time $t$ (measured using the trapezoidal rule) and expressed as a percentage of the area of the rectangle described by $100 \%$ dissolution in the same time. $D E$ results were submitted to analysis of variance (one-way ANOVA) and Student's $t$-test employing STATISTICA 7 software (StatSoft, USA). Scheffé's test was applied to determine differences between the means obtained. Differences between means were considered statistically significant at $p<0.05$. Mathematical models are showed in Table 2 .

\section{RESULTS AND DISCUSSION \\ Development of the Dissolution Test}

As expected for a poorly water-soluble drug, the product exhibited poor dissolution in buffered solutions with different $\mathrm{pH}$ values (data not shown). Since rimonabant is a weakly basic compound with a $\mathrm{p} K_{\mathrm{a}}$ of $3.6(18)$, its dissolution in $0.1 \mathrm{M}$ hydrochloric acid was substantially higher due to its protonation in low $\mathrm{pH}$ values, which increases its solubility. In this medium, a maximum dissolution of $60.63 \%$ was achieved within $60 \mathrm{~min}$, and saturation of the medium seems to occur in 30 min of the test. Increased dissolution was obtained with the surfactant agent SLS in ultrapure 


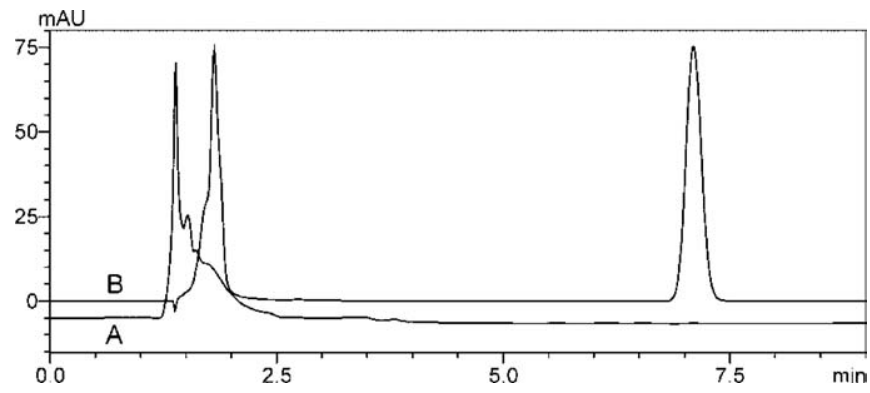

Figure 1. Specificity test for rimonabant in dissolution medium. Chromatograms of (A) placebo and (B) reference tablet formulation after 60 min of the dissolution test.

water, which was added to the medium to improve the solubility of the drug. Surfactants enhance the dissolution rate of poorly water-soluble drugs, even when present at a level below the critical micellar concentration (CMC). This may be due to a reduction in the interfacial tension as well as a possible association between the drug, excipients, or both and the surfactants (19). The concentration of SLS used in the medium is below its CMC, $8.2 \mathrm{mM}$ or $0.24 \%$ in water at $20^{\circ} \mathrm{C}(15)$, which probably resulted in a solubilization by the tensoative effect in the particle-medium layer and not by micellar solubilization. From the initial screening result, the conditions selected were $900 \mathrm{~mL} 0.15 \%$ SLS in water as dissolution medium and USP Apparatus 2 (paddle) at $50 \mathrm{rpm}$, which were used in all subsequent tests.

\section{HPLC Assay}

An analytical method was developed to analyze the dissolution of immediate-release tablets and capsules. The standard curve used for the determination of drug in samples showed excellent correlation in the concentration range of $0.5-50 \mu \mathrm{g} / \mathrm{mL}\left(y=86036 x+464.65, r^{2}=1\right)$. The limit of quantification of the assay was $0.24 \mu \mathrm{g} / \mathrm{mL}$, and the time of analysis was 9 min per sample. Chromatographic peak purity values obtained by DAD were greater than 0.9999, indicating homogenous peaks and absence of any coeluting peak with the same retention time (7.1 min). As seen in Figure 1, no interferences from excipients or dissolution medium with the peak of interest were observed through the analysis of a placebo formulation, confirming the selectivity of the method.

\section{Drug Stability}

Samples remained stable in dissolution medium for at least $24 \mathrm{~h}$ when maintained at room temperature and protected from light. The concentration found after storage at $25^{\circ} \mathrm{C}$ for $24 \mathrm{~h}$ was $100.7 \%$ of the initial drug amount, indicating that samples can be analyzed on the day after the assay, because there is no significant change in the drug content. Peak purity of rimonabant remained within high values, which was assessed by DAD analysis. No degrada-

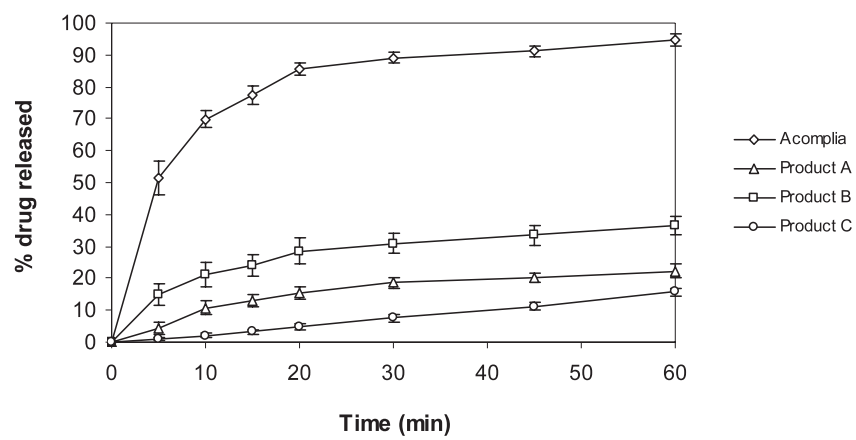

Figure 2. Dissolution profiles of rimonabant (mean $\pm S D, n=12$ ) from the reference tablet formulation (Acomplia) and compounded capsules obtained from different pharmacies (products $A, B$, and C). Dissolution medium $0.15 \%$ SLS in water $\left(900 \mathrm{~mL}\right.$ ) at $37^{\circ} \mathrm{C}$, USP Apparatus 2 (paddle), stirring speed $50 \mathrm{rpm}$.

tion product or decrease in peak area of rimonabant was observed, indicating that the drug is stable in dissolution samples through the period evaluated.

\section{In Vitro Dissolution from Different Solid Dosage Forms}

Figure 2 demonstrates the dissolution profiles of the poorly soluble drug rimonabant from different commercially available formulations. Acomplia film-coated tablets showed rapid disintegration and a fast dissolution rate (more than $50 \%$ of the drug was released within $5 \mathrm{~min}$ and about $95 \%$ was dissolved after $60 \mathrm{~min}$ ). The relative standard deviation (RSD) value was $10.29 \%$ at the early time point ( $5 \mathrm{~min}$ ) and ranged from $1.65 \%$ to $3.89 \%$ at the other time points. The dissolution profiles were analyzed by model-dependent methods. The selection of model was based on the best correlation coefficient, model selection criteria, and graphic adjustment. As seen in Table 3, the dissolution profile best fits the bi-exponential model due to higher values for $r$ and MSC compared with those obtained with the mono-exponential model. For Acomplia, results from mathematical modeling demonstrate that about $74 \%$ of the drug is released in a fast initial phase $\left(0.2040 \mathrm{~min}^{-1}\right)$, followed by $26 \%$ at a slower rate $\left(0.0265 \mathrm{~min}^{-1}\right)$, which is about 10 -fold lower than $k_{1}$. For product A, 20\% is released at a faster rate $\left(0.0667 \mathrm{~min}^{-1}\right)$ followed by $80 \%$ at a slower rate $\left(0.0005 \mathrm{~min}^{-1}\right)$. The product $B$ profile is also better described by the bi-exponential model. Although product $C$ also presented a higher value for MSC, the graphic shows a dissolution profile compatible with zero-order kinetics in the interval 0-60 min, which is characteristic of controlled-release formulations (20). This probably occurred due to high concentration of HPMC in the formulation. To prove our hypothesis, modeling was done using a zero-order model. In this case, the observed rate constant $k$ was $0.2530 \mathrm{~min}^{-1}(r=0.9990, \mathrm{MSC}=5.0006)$.

Marked differences in the kinetics of drug release can be observed in Figure 2. Compounded capsules (products 
Table 3. Observed Rate Constants, Correlation Coefficients, and MSC Obtained by Fitting of Rimonabant Release from Different Dosage Forms

\begin{tabular}{lcccc}
\hline & Acomplia & Product A & Product B & Product C \\
\hline Mono-exponential & \multicolumn{3}{c}{} & \\
\hline$k\left(\mathrm{~min}^{-1}\right)$ & $0.1133 \pm 0.0109$ & $0.0055 \pm 0.0007$ & $0.0107 \pm 0.0017$ & $0.0027 \pm 0.0001$ \\
\hline$r($ range $)$ & 0.9914 & 0.9206 & 0.8864 & 0.9980 \\
\hline MSC (range) & 3.0340 & 0.9290 & 0.3301 & 4.3777 \\
\hline Bi-exponential & & & & \\
\hline$k_{1}\left(\mathrm{~min}^{-1}\right)$ & $0.2040 \pm 0.0232$ & $0.0667 \pm 0.0234$ & $0.1517 \pm 0.0217$ & $0.0969 \pm 0.0528$ \\
\hline$k_{2}\left(\mathrm{~min}^{-1}\right)$ & $0.0265 \pm 0.0066$ & $0.0005 \pm 0.0012$ & $0.0028 \pm 0.0005$ & $0.0031 \pm 0.0001$ \\
\hline $\mathrm{A}(\mu \mathrm{g} / \mathrm{mL})$ & $0.7414 \pm 0.0550$ & $0.2024 \pm 0.0592$ & $0.2463 \pm 0.0181$ & $-0.0171 \pm 0.0064$ \\
\hline$B(\mu \mathrm{g} / \mathrm{mL})$ & $0.2570 \pm 0.0541$ & $0.8018 \pm 0.0012$ & $0.7521 \pm 0.0173$ & $1.0173 \pm 0.0065$ \\
\hline$r(\mathrm{range})$ & 0.9993 & 0.9967 & 0.9986 & 0.9998 \\
\hline $\mathrm{MSC}(\mathrm{range})$ & 5.5469 & 4.0224 & 4.8659 & 6.6435 \\
\hline
\end{tabular}

$A, B$, and C) reached discrete and unsatisfactory rates and extent of dissolution when compared with the reference product. The differences between the products could change their bioavailability. Some reasons for this poor performance could be the choice of excipients, source of raw material and process of obtaining it, polymorphism, the presence of freebase versus the more soluble salt forms, particle size of active substance, and dosage form manufacturing process. It is known that Acomplia tablets are manufactured by a standard wet granulation process, and this factor may be related to the difference in dissolution profiles of rimonabant relative to the drug formulated in capsule dosage form (9). Polymorphism of drug substance may also be related to different dissolution characteristics, since polymorphs have distinct crystalline structures; two polymorphic forms of rimonabant were identified during development (9). The effect of particle shape on the dissolution rate of poorly soluble drugs had also been reported (21).

To understand further the differences in the dissolution profiles of different dosage forms, five different formulations of 20-mg capsules were prepared. The release of poorly soluble rimonabant depended on the water solubility of diluent. Both the rate and extent of dissolution of drug from lactose-based formulations $(3,4$, and 5$)$ were significantly higher than from starch-based formulations 1 and 2 (Figure 3). The addition of $2 \%$ (w/w) SLS (formulation 2 versus formulation 1) to starch-based formulations enhanced the rate of release during the early time points (30 min), although percent release was almost equal after $60 \mathrm{~min}$. This can be attributed to the capacity of surfactants to facilitate the wettability of the powder, allowing the dissolution fluid to permeate the solid particles more efficiently, thus increasing the surface area exposed to the medium and enhancing the dissolution rate of the drug
(6). The effect of SLS was not observed in formulations containing lactose as diluent. According to the literature (22), the dissolution of hard gelatin capsule shells in $50 \mathrm{mM}$ sodium phosphate buffer $\mathrm{pH} 6.8$ was not affected by adding $1 \%$ SLS to the medium. However, SLS in the dissolution medium caused a delay in the dissolution of hard gelatin capsule shells at $\mathrm{pH}<5$ due to the formation of a less-soluble precipitate of gelatin. Since the $\mathrm{pH}$ used in this test method is close to 7, we attributed the effect of SLS to its capacity of increasing the amount of powder surface available to the medium, and not to the increase of the dissolution of the gelatin capsule shell. In addition to the hydrophilic diluent lactose, the disintegrants crospovidone and croscarmellose sodium could also contribute to the higher drug release from formulations 3,4 , and 5 relative to starch-based formulations. The maximum dissolved fraction obtained in this study occurred with formulation 5 (69.09\% within $60 \mathrm{~min})$.

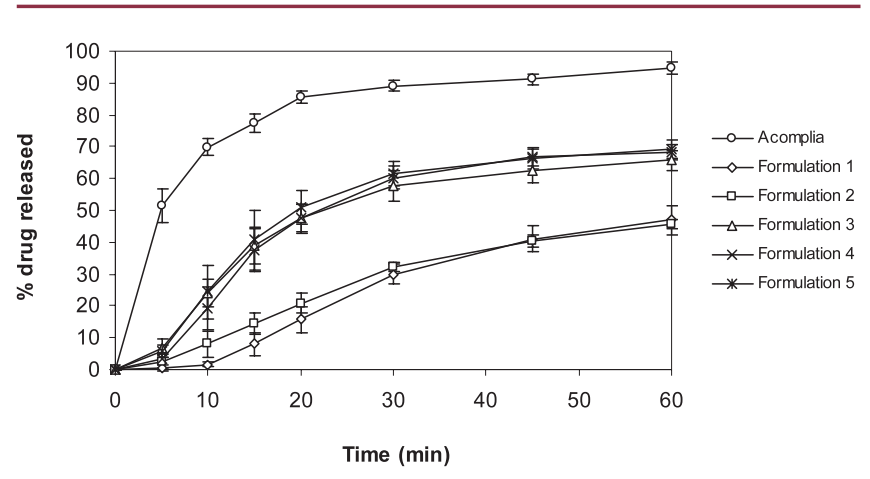

Figure 3. In vitro release study of rimonabant $(n=6)$ after formulation modifications and particle size reduction. Conditions are described in Figure 2. 
Table 4. Comparison of Dissolution Profiles of Rimonabant from 20-mg Solid Oral Dosage Forms through Dissolution Efficiency (DE)

\begin{tabular}{lc}
\hline Formulation & DE\% (mean \pm SD) \\
\hline Acomplia & $80.46 \pm 1.54$ \\
\hline Product A & $15.98 \pm 1.12$ \\
\hline Product B & $27.93 \pm 3.08$ \\
\hline Product C & $7.44 \pm 0.93$ \\
\hline Formulation 1 & $25.76 \pm 3.12$ \\
\hline Formulation 2 & $27.02 \pm 1.72$ \\
\hline Formulation 3 & $47.65 \pm 3.79$ \\
\hline Formulation 4 & $48.67 \pm 3.03$ \\
\hline Formulation 5 & $50.36 \pm 2.99$ \\
\hline
\end{tabular}

Because particle size may affect the solubility of the drug and therefore the dissolution rate of the product, particle size reduction is an approach commonly used to enhance dissolution of poorly water-soluble drugs and thereby affect bioavailability. Particle size reduction was accomplished by milling the drug and mixing it with lactose, disintegrants, and surfactant (formulation 5 versus formulation 3). Particle size was reduced from 53 to $25 \mu \mathrm{m}$ (2-fold reduction) by the process employed. Micronization of rimonabant to a mean particle size of $25 \mu \mathrm{m}$ did not enhance either the rate or extent of release when compared with untreated drug, as seen in Figure 3. It is well established in literature that particle size reduction to 5-10 $\mu \mathrm{m}$ generally causes an increase in dissolution for poorly soluble drugs, as shown for the antifungal griseofulvin (5), antiplatelet cilostazol (23), and steroidal hormone danazol (24). It can therefore suggest that the process employed was not suitable for particle size reduction, and an increase in micronization will possibly enhance the in vitro dissolution of rimonabant by increasing the surface area that is exposed to the medium.

$D E \%$ was calculated for all products and formulations (Table 4). One-way ANOVA of the DE\% values showed that the percent drug released is not similar for the commercially available products $(p<0.05)$. For the formulations used in the vitro release study, Scheffé's test showed that starch-based formulations are significantly different from lactose-based formulations. The $D E \%$ of formulation 2 does not differ from formulation 1, and formulations 3,4 and 5 are not significantly different from one other. These data confirm the previous results involving the addition of surfactant to the formulation and reducing the particle size of the drug.

\section{CONCLUSION}

A dissolution test was developed for rimonabant tablets and hard gelatin capsules according to USP 32 (16). The conditions selected for the test method are $900 \mathrm{~mL}$ of
$0.15 \%$ SLS in water as dissolution medium, USP Apparatus 2 (paddle), a stirring speed of $50 \mathrm{rpm}$, and drug analysis by HPLC. The proposed dissolution method was then successfully applied to investigate the release profile of rimonabant from solid oral dosage forms. Dissolution profiles were analyzed using model-dependent (curve-fitting) and independent $(D E)$ approaches. The kinetics of drug release from Acomplia was better described by the biexponential model. For the five laboratory formulations, our study has shown that deliberate changes in the excipients had a marked affect on rimonabant dissolution. There are many variables influencing the in vitro dissolution of Class 2 drugs from immediate-release solid oral dosage forms. Differences in the type of salt used (rimonabant hydrochloride versus rimonabant base in Acomplia), dosage form (capsule versus film-coated tablet), manufacturing process (capsule filling versus wet granulation), and distinct particle size distribution of bulk drug are the main factors influencing the extent of dissolution from prepared formulations. Of the commercially available dosage forms, only the reference product showed dissolution of up to $85 \%$ within $60 \mathrm{~min}$, indicating a need to improve the formulations of the other products. Therefore, the manipulation of Class 2 drugs associated with an incorrect choice of excipients or problems during the formulation may be risks to the quality of these products, compromising their therapeutic efficacy.

\section{ACKNOWLEDGMENTS}

The authors wish to thank CNPq (Conselho Nacional de Desenvolvimento Científico e Tecnológico) and FAPERGS (Fundação de Amparo à Pesquisa do Estado do Rio Grande do Sul) for their support. F. K. Hurtado thanks CAPES (Coordenação de Aperfeiçoamento de Pessoal de Nível Superior) for his fellowship.

\section{REFERENCES}

1. Varshosaz, J.; Talari, R.; Mostafavi, S. A.; Nokhodchi, A. Dissolution enhancement of gliclazide using in situ micronization by solvent change method. Powder Technol. 2008, 187 (3), 222-230.

2. Lima, D. E.; Santos, L. D.; Lima, E. M. Stability and in vitro release profile of enalapril maleate from different commercially available tablets: possible therapeutic implications. J. Pharm. Biomed. Anal. 2008, 47 (4-5), 934-937.

3. Dressman, J. B.; Reppas, C. In vitro-in vivo correlations for lipophilic, poorly water-soluble drugs. Eur. J. Pharm. Sci. 2000, 11 (2), S73-S80.

4. Vogt, M.; Kunath, K.; Dressman, J. B. Dissolution improvement of four poorly water soluble drugs by cogrinding with commonly used excipients. Eur. J. Pharm. Biopharm. 2008, 68 (2), 330-337.

5. Hörter, D.; Dressman, J. B. Influence of physicochemical properties on dissolution of drugs in the gastrointestinal tract. Adv. Drug Deliv. Rev. 2001, 46 (1-3), 75-87. 
6. Aulton, M. E. Pharmaceutics: The Science of Dosage Form Design, 2nd ed.; Churchill Livingstone: New York, 2001.

7. Dissolution Testing of Immediate Release Solid Oral Dosage Forms; Guidance for Industry; U.S. Department of Health and Human Services, Food and Drug Administration, Center for Drug Evaluation and Research (CDER), U.S. Government Printing Office: Washington, DC, 1997.

8. Pouton, C. W. Formulation of poorly water-soluble drugs for oral administration: Physicochemical and physiological issues and the lipid classification system. Eur. J. Pharm. Sci. 2006, 29 (3-4), 278-287.

9. Acomplia: Scientific Discussion, EMEA/H/C/666; European Public Assessment Report; European Medicines Agency: London, 2006.

10. Hurtado, F. K.; Ravanello, A.; Arend, M. Z.; Wrasse, M.; Dalmora, S. L.; Rolim, C. M. B. Stability-indicating RPHPLC method for the determination of rimonabant in a pharmaceutical dosage form. J. AOAC Int. 2010, 93 (3), 869-875.

11. Papp, R.; Luk, P.; Mullett, W. M.; Kwong, E.; Debnath, S.; Thibert, R. Development of a discriminating in vitro dissolution method for a poorly soluble NO-donating selective cyclooxygenase-2 inhibitor. J. Pharm. Biomed. Anal. 2008, 47 (1), 16-22.

12. Noory, C.; Tran, N.; Ouderkirk, L.; Shah, V. Steps for development of a dissolution test for sparingly water-soluble drug products. Dissolution Technol. 2000, 7 (1), 16.

13. Shah, V. P.; Noory, A.; Noory, C.; McCullough, B.; Clarke, S.; Everett, R.; Naviasky, H.; Srinivasan, B. N.; Fortman, D.; Skelly, J. P. In vitro dissolution of sparingly watersoluble drug-dosage forms. Int. J. Pharm. 1995, 125 (1), 99-106.

14. International Conference on Harmonisation. Validation of Analytical Procedures: Text and Methodology, Q2(R1); ICH Harmonized Tripartite Guideline; Geneva, 2005.

15. Rowe, R. C. Handbook of Pharmaceutical Excipients, 5th ed.; The Pharmaceutical Press: London, 2006.
16. The United States Pharmacopeia and National Formulary USP 32-NF 27; The United States Pharmacopeial Convention, Inc.: Rockville, MD, 2009.

17. Yuksel, N.; Kanik, A. E.; Baykara, T. Comparison of in vitro dissolution profiles by ANOVA-based, modeldependent and -independent methods. Int. J. Pharm. 2000, 209 (1-2), 57-67.

18. Bergström, C. A. S.; Wassvik, C. M.; Johansson, K.; Hubatsch, I. Poorly soluble marketed drugs display solvation limited solubility. J. Med. Chem. 2007, 50 (23), 5858-5862.

19. Shah, V. P.; Konecny, J. J.; Everett, R. L.; McCullough, B.; Noorizadeh, A. C.; Skelly, J. P. In vitro dissolution profiles of water-insoluble drug-dosage forms in the presence of surfactants. Pharm. Res. 1989, 6 (7), 612-618.

20. Chattaraj, S. C.; Das, S. K. Effect of formulation variables on dissolution profile of diclofenac sodium form ethyland hydroxypropyl methylcellulose tablets. Drug Dev. Ind. Pharm. 1996, 22 (7), 555-559.

21. Mosharraf, M.; Nyström, C. The effect of particle size and shape on the surface specific dissolution rate of microsized practically insoluble drugs. Int. J. Pharm. 1995, 122 (1-2), 35-47.

22. Zhao, F.; Malayev, V.; Rao, V.; Hussain, M. Effect of sodium lauryl sulfate in dissolution media on dissolution of hard gelatin capsule shells. Pharm. Res. 2004, 21 (1), 144-148.

23. Liversidge, G. G.; Cundy, K. C. Particle size reduction for improvement of oral bioavailability of hydrophobic drugs: I. Absolute oral bioavailability of nanocrystalline danazol in beagle dogs. Int. J. Pharm. 1995, 125 (1), 91-97.

24. Jinno, J.; Kamada, N.; Miyake, M.; Yamada, K.; Mukai, T.; Odomi, M.; Toguchi, H.; Liversidge, G. G.; Higaki, K.; Kimura, T. Effect of particle size reduction on dissolution and oral absorption of a poorly water-soluble drug, cilostazol, in beagle dogs. J. Control. Release 2006, 111 (1-2), 56-64. 